\title{
Epiteliopatía corneal por alteración sensitiva: queratitis neurotrófica
}

\section{Corneal epitheliopathy due to sensitive impairment: Neurotrophic keratopathy}

\author{
Alejandro Babayán-Sosa* y Oscar Baca-Lozada \\ Fundación Hospital Nuestra Señora de la Luz, IAP, Ciudad de México, México
}

\begin{abstract}
Resumen
La queratitis neurotrófica es una enfermedad degenerativa corneal caracterizada por la reducción en la sensibilidad, una desepitelización y una reparación corneal ineficiente. Se han descrito muchas causas de queratitis neurotrófica, como la queratitis herpética, la diabetes, un trauma y las cirugías oftálmicas y neurológicas, que tienen en común el daño al nervio trigémino. El diagnóstico requiere un interrogatorio exhaustivo de los antecedentes oculares y sistémicos, un examen oftalmológico completo y la valoración de la sensibilidad corneal. La queratitis neurotrófica se clasifica de acuerdo al daño que se observa: queratitis punteada superficial (estadio I), defecto epitelial persistente (estadio II) y perforación y lisis (estadio III). El tratamiento se basa en la gravedad y está dirigido a promover la reepitelización y evitar la progresión de la enfermedad a lisis y perforación. Actualmente no existen tratamientos médicos específicos y los procedimientos quirúrgicos, como el injerto de membrana amniótica y el colgajo conjuntival, son efectivos para conservar la integridad del ojo, sin que se mejore la sensibilidad corneal o la visión.
\end{abstract}

Palabras clave: Queratitis neurotrófica. Sensibilidad corneal. Inervación corneal. Defecto epitelial persistente. Nervio trigémino.

\begin{abstract}
Neurotrophic keratitis is a degenerative disease characterized by corneal sensitivity reduction, spontaneous epithelium breakdown, and impairment of corneal healing. Several causes of neurotrophic keratitis, including herpetic keratitis, diabetes, trauma and ophthalmic and neurosurgical procedures, share the common mechanism of trigeminal damage. Diagnosis requires accurate investigation of clinical ocular and systemic history, complete eye examination, and assessment of corneal sensitivity. Neurotrophic keratitis can be classified according to severity of corneal damage, superficial punctate keratitis (stage 1), persistent epithelial defect (stage 2), and perforation and melting (stage 3). Management should be based on clinical severity, and aimed at promoting corneal healing and preventing progression of the disease to stromal melting and perforation. Currently, no specific medical treatment exists, and surgical approaches, such as amniotic membrane transplantation and conjunctival flap, are effective in preserving eye integrity, without ameliorating corneal sensitivity or visual function.
\end{abstract}

Key words: Neurotrophic keratitis. Cornea sensitivity. Cornea innervation. Persistent epithelial defect. Trigeminal nerve.

Correspondencia:

*Alejandro Babayán-Sosa

Col. Tabacalera, Del. Cuauhtémoc Fecha de recepción: 04-04-2017

Disponible en internet: 14-05-2018

C.P. 06030, Ciudad de México, México Fecha de aceptación: 01-07-2017

Rev Mex Oftalmol. 2018;92(3):117-122

E-mail: alejandro.babayan@gmail.com DOI: 10.24875/RMO.M18000018 www.rmo.com.mx

0187-4519/@ 2017 Sociedad Mexicana de Oftalmología. Publicado por Permanyer México. Este es un artículo Open Access bajo la licencia CC BY-NC-ND (http://creativecommons.org/licenses/by-nc-nd/4.0/). 


\section{Introducción}

La queratitis neurotrófica o queratopatía neurotrófica es una enfermedad degenerativa poco frecuente que resulta de una afectación de la sensibilidad corneal, asociada a defectos epiteliales, úlcera corneal, defectos en la cicatrización, lisis corneal y perforación ${ }^{1}$. Se ha descrito la queratitis neurotrófica tanto primaria (idiopática) como secundaria a varias enfermedades oculares y sistémicas. La lesión de la inervación corneal trigeminal es el mecanismo patogénico habitual. Muchas enfermedades oculares y neurológicas pueden provocar una lesión en diferentes puntos de la inervación corneal: el tracto trigeminal, el ganglio o las fibras sensitivas. La causa más frecuente de anestesia corneal en nuestro país es la infección viral (queratitis por herpes simple y herpes zóster), seguida de las quemaduras químicas, las heridas físicas y la cirugía corneal. Lesiones intracraneales como los neurinomas, los meningiomas y los aneurismas también pueden determinar una compresión en el nervio trigeminal o el ganglio y producir un daño en la sensibilidad corneal. Enfermedades sistémicas como la diabetes, la esclerosis múltiple y la lepra pueden disminuir la función sensitiva 0 dañar directamente las fibras sensitivas, conduciendo a la anestesia corneal2-8. El diagnóstico clínico se orienta fácilmente a partir de los antecedentes, pero el manejo de esta condición es uno de los retos más difíciles y desafiantes de todas las enfermedades corneales.

\section{Fisiopatología}

La queratitis por exposición es un término más apropiado para la queratitis asociada a la parálisis del séptimo. El termino queratitis neurotrófica debe reservarse para el daño corneal secundario a las lesiones del trigémino. La córnea contiene una de las inervaciones más ricas de todos los tejidos corporales. Los nervios sensitivos corneales juegan un papel clave en el mantenimiento de la integridad anatómica y la función de la córnea, particularmente del epitelio. En el caso de la parálisis del quinto nervio, es la pérdida de sensibilidad corneal lo que produce una disminución del parpadeo ocasionando defectos epiteliales y evaporación de la película lagrimal, desecación del epitelio corneal por exposición. La falta de parpadeo resulta en una vasodilatación del borde palpebral y de los vasos perilímbicos, así como en la presencia de edema. El daño epitelial corneal y conjuntival en la zona de la apertura palpebral se hace evidente y posteriormente puede acompañarse de iritis, que se manifiesta como flare y Tyndall, en la cámara anterior. La membrana celular epitelial se daña con la subsecuente muerte celular por cambios osmolares. Clínicamente ocurren múltiples y pequeños defectos epiteliales en la zona de exposición, que posteriormente aumentan de tamaño. Se presenta una hiperplasia epitelial como mecanismo de compensación por la irregularidad de la superficie en diferentes áreas de la córnea. La mitosis celular disminuye su velocidad y se reduce la velocidad de migración celular. Inicialmente los defectos son rellenados, pero una vez que existe lesión en la capa de Bowman no se logra llenar esos defectos (superficie estromal irregular que no permite el deslizamiento y adhesión de las células), lo que da un aspecto de aumento del grosor epitelial. El término ulceración puede considerarse desde el momento en que se pierden todas las capas epiteliales, incluyendo la membrana basal, dejando expuesta la capa de Bowman o bien queda expuesto el estroma por la pérdida de esta última. La presencia de neovasos superficiales está condicionada después de cierto tiempo y se involucran la pérdida celular, el edema, la inflamación y la hipoxia, con ulceraciones epiteliales, estromales, en ocasiones infecciones y perforaciones. La ulceración estromal resulta de la degradación de enzimas proteolíticas y colagenolíticas. Dichas enzimas pueden ser elaboradas por células epiteliales dañadas, células conjuntivales, fibroblastos y células inflamatorias ${ }^{9}$. Varios estudios han demostrado que la pérdida de la inervación sensitiva corneal lleva a una disminución en el metabolismo y mitosis de las células epiteliales y, consecuentemente, a un fallo epitelial. Varios estudios se han centrado en el papel de los neuromediadores sensitivos en la fisiopatología del epitelio corneal. Dichos estudios han demostrado la depleción de sustancia P (SP) y acetilcolina (Ach) en la córnea de rata después de una lesión de los nervios sensitivos in vitro, la SP, el péptido relacionado con el gen de la calcitonina (CGRP) y la $\mathrm{Ach}^{10,11}$. Chikama, et al. lograron una recuperación completa de una queratitis neurotrófica en un paciente tratado con una combinación de colirios de SP e insulin-like growth factor-1 (IGF-1) 12 . Infirieron que la combinación de SP e IGF-1 estimula la migración de células epiteliales corneales y la expresión de integrina $\alpha 5$ y $\beta 1$, esencial para la adhesión de las células epiteliales a las proteínas de la matriz extracelular. La evidencia clínica y experimental sugiere un control bidireccional de la proliferación epitelial corneal: Ios neuromediadores sensitivos promueven la mitosis de las células epiteliales 
mientras que, por otro lado, los mediadores simpáticos, adrenalina y noradrenalina, la reducen.

\section{Epidemiología}

Hasta nuestro conocimiento no hay en la literatura ningún dato acerca de la epidemiología de la queratitis neurotrófica. Se estima una prevalencia menor a $5 / 10,00$ individuos. Cuando analizamos la prevalencia de las principales causas de queratitis neurotrófica, encontramos que el $6 \%$ de las queratitis por herpes simple desarrollan este padecimiento, así como el $12.8 \%$ de las queratitis por herpes zóster y el $2.8 \%$ de los pacientes sometidos a cirugía por neuralgia del trigémino ${ }^{13-16}$.

\section{Causas}

Las principales causas oculares la queratitis herpética (simple y zóster), las quemaduras, el trauma, las distrofias y algunos medicamentos tópicos. Sistémicamente las causas de daño trigeminal son iatrogénicas, tumorales, esclerosis múltiple, diabetes, lepra, medicamentos y síndromes congénitos. El listado completo se describe en la tabla 1.

\section{Diagnóstico}

El paso esencial en el diagnóstico de una queratitis neurotrófica es llevar a cabo un interrogatorio exhaustivo de la historia médica y quirúrgica para poder determinar la causa de la hipoestesia corneal. Enfermedades sistémicas como la diabetes mellitus o las terapias sistémicas (neurolépticos, antipsicóticos y antihistamínicos) pueden lesionar la inervación corneal trigeminal. La cirugía o los traumatismos corneales, el abuso de anestésicos tópicos, los medicamentos tópicos crónicos, las quemaduras químicas o el abuso de las lentes de contacto pueden dañar localmente los nervios sensitivos corneales. Las neoplasias cerebrales y los accidentes vasculares pueden comprometer el quinto par craneal o su núcleo. Puede ocurrir una lesión del séptimo y del quinto pares craneales en una oftalmopatía tiroidea, una neoplasia periocular infiltrante 0 tras la resección quirúrgica de un neurinoma del acústico. Los aneurismas o enfermedad del seno cavernoso pueden causar alteraciones de la motilidad ocular, mientras que una lesión orbitaria intraconal puede estar asociada a alteraciones del nervio óptico y/o anomalías pupilares. La presencia de signos neurológicos requiere un manejo conjunto con el
Tabla 1. Causas de queratitis neurotrófica

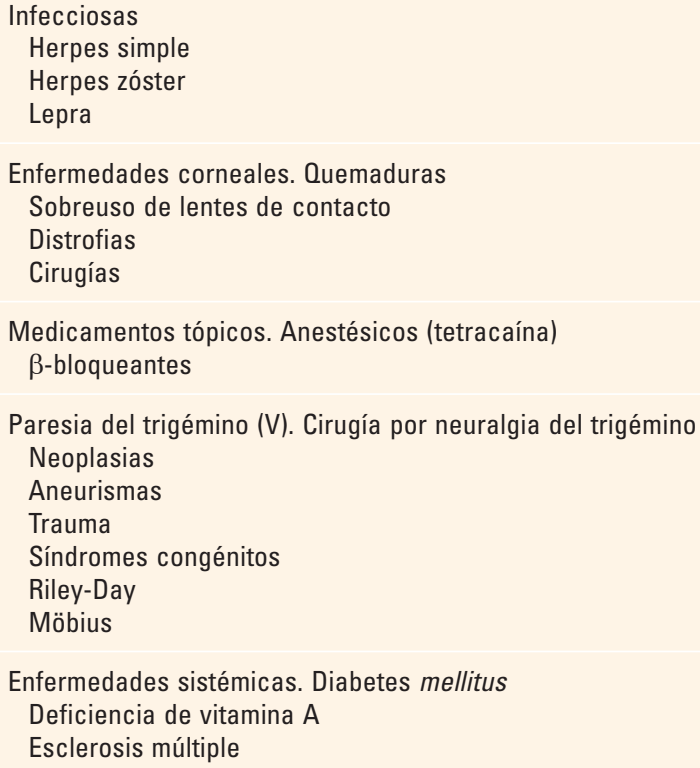

Enfermedades corneales. Quemaduras Sobreuso de lentes de contacto Distrofias

Cirugías

Medicamentos tópicos. Anestésicos (tetracaína) $\beta$-bloqueantes

Paresia del trigémino (V). Cirugía por neuralgia del trigémino Neoplasias

Aneurismas

Trauma

Síndromes congénitos

Riley-Day

Möbius

Enfermedades sistémicas. Diabetes mellitus Deficiencia de vitamina A

Esclerosis múltiple

neurólogo. Se debe llevar a cabo un examen oftalmológico completo. La prueba de sensibilidad corneal debe realizarse tocando la córnea central y periférica con la punta de un hisopo de algodón. Como alternativa, puede utilizarse el estesiómetro de Cochet-Bonnet para localizar y cuantificar la pérdida de sensibilidad corneal, registrando la respuesta del paciente al tacto de un hilo de nailon (de entre 0 y $6 \mathrm{~cm}$ ). Estas técnicas no permiten la identificación de los valores exactos de la sensibilidad corneal normal debido a los métodos de medida y a la gran variabilidad de la sensibilidad corneal (depende de la edad, el sexo, el área examinada y la hora del día). Generalmente la gravedad de la queratitis neurotrófica está relacionada con la de la afección de la sensibilidad corneal. Debe realizarse la prueba de Schirmer, ya que la producción de lágrima puede estar afectada por la reducción de la sensibilidad corneal. La tinción con fluoresceína, rosa de Bengala o verde de lisamina muestra cambios epiteliales corneales y conjuntivales. El examen del margen palpebral, su posición y motilidad, es importante, ya que una queratitis de exposición y una blefaritis pueden estar asociadas a una queratitis neurotrófica. Deben examinarse las pupilas y los nervios craneales en la búsqueda de signos sugestivos de enfermedad intracraneal ${ }^{17-19}$.

\section{Diagnósticos diferenciales}

La queratopatía punteada superficial y las anomalías de la película lagrimal, que caracterizan el estadio I de 
la enfermedad, se observan también frecuentemente en otras enfermedades oculares tales como el ojo seco, la queratitis por exposición, la toxicidad por medicamentos tópicos y el sobreuso de lentes de contacto. La presencia de síntomas oculares como ardor, sensación de cuerpo extraño, fotofobia y ojo seco orientan el diagnóstico hacia otras enfermedades de la superficie ocular; lo característico de la queratitis neurotrófica es la anestesia corneal. También puede verse una vascularización corneal superficial y un defecto epitelial en la insuficiencia de células del limbo. En este caso, la citología de impresión puede permitir la identificación del fenotipo epitelial mediante la inmunohistoquímica para citoqueratinas (el epitelio corneal es citoqueratina 3 positivo y citoqueratina 19 negativo, mientras que el epitelio conjuntival es citoqueratina 19 positivo y citoqueratina 3 negativo ${ }^{20}$. Sin embargo, las enfermedades que causan insuficiencia límbica pueden dañar los nervios corneales sensitivos y causar también una queratitis neurotrófica. Las úlceras infecciosas, tóxicas o inmunes siempre presentan una inflamación ocular importante, infiltrados estromales y síntomas. Siempre se requieren exámenes microbiológicos para bacterias, hongos y virus; las úlceras corneales postherpéticas pueden estar asociadas a una infección viral activa. Cualquier tratamiento local debe suspenderse para excluir úlceras corneales tóxicas y asimismo debe considerarse una evaluación sistémica para detectar alteraciones inmunes.

\section{Clasificación}

El estadio I se caracteriza por una queratopatía punteada, hiperplasia e irregularidad epitelial, neovascularización superficial y cicatrización estromal. El estadio II está caracterizado por la presencia de un defecto epitelial persistente, más frecuentemente localizado en la mitad superior de la córnea. Habitualmente, alrededor del defecto epitelial hay un área de epitelio pobremente adherido, opaco y edematoso, que puede despegarse espontáneamente y conducir a un aumento del tamaño del defecto. La cicatrización epitelial es inadecuada y los bordes del defecto se enrollan. También se pueden observar pliegues en la membrana de Descemet y un edema estromal con una reacción inflamatoria en la cámara anterior; puede aparecer, raramente, un hipopión estéril. El estadio III se caracteriza por el compromiso estromal, con una úlcera corneal que puede progresar a perforación y/o lisis estromal. El resumen de los estadios se encuentra en la tabla 2.
Tabla 2. Clasificación de Mackie

\author{
Estadio I. Queratitis punteada superficial \\ Disminución del TRL \\ Tinción conjuntival inferior \\ Cicatrización \\ Estadio II. Defecto epitelial \\ Edema corneal \\ Epitelio suelto \\ Estadio III. Úlcera persistente \\ Lisis estromal \\ Perforación
}

\section{Tratamiento}

Los objetivos del tratamiento de la queratitis neurotrófica son prevenir la progresión del daño corneal y mejorar la transparencia de la córnea. La terapia debe instaurarse de forma temprana y con base en el estadio clínico de la enfermedad. En el estadio I la terapia trata de mejorar la calidad epitelial y la transparencia, y evitar el defecto epitelial. Es obligatorio suspender toda medicación tópica, de ser posible, y considerar los posibles efectos adversos de terapias sistémicas como neurolépticos, antipsicóticos y antihistamínicos. Las lágrimas artificiales libres de conservadores pueden ayudar a mejorar la superficie corneal. Se han utilizado lentes de contacto terapéuticas corneales o esclerales, pero su uso puede incrementar el riesgo de infecciones secundarias y causar un hipopión estéril, por lo que debe limitarse ${ }^{21}$. Otras enfermedades de la superficie corneal como la queratitis de exposición, el ojo seco y la insuficiencia límbica, si están asociadas a la queratitis neurotrófica, empeoran el pronóstico de la enfermedad y requieren un tratamiento específico. En estos casos es importante corregir la disfunción palpebral y considerar un trasplante de células límbicas. En el estadio II los objetivos del tratamiento son evitar el desarrollo de la úlcera corneal, promover la cicatrización del defecto epitelial y prevenir la recurrencia de la desepitelización. Se requiere siempre la suspensión de todos los medicamentos tópicos, y se puede mejorar la superficie corneal mediante el uso de lágrimas artificiales sin conservadores. Los pacientes deben ser monitorizados cuidadosamente porque la progresión de la enfermedad frecuentemente es asintomática. En caso de úlceras corneales que no responden a las lágrimas artificiales o a lentes de contacto terapéuticas, la tarsorrafia es el procedimiento más utilizado y simple. Si se consigue la curación, la apertura de la tarsorrafia puede realizarse tras unas pocas semanas, pero abrir la tarsorrafia prematuramente puede resultar en una recurrencia del 
defecto epitelial corneal ${ }^{22}$. Como alternativa, es posible cubrir el defecto epitelial mediante un injerto de membrana amniótica o utilizar una pesa palpebral o una inyección de toxina botulínica $A$ en el elevador del párpado $^{23}$. En el estadio III la terapia va dirigida a promover la curación corneal y prevenir la lisis corneal y la perforación. Al igual que en los estadios I y II, es importante evitar el efecto tóxico de los tratamientos tópicos, de ahí que sólo se sugiera el uso de lágrimas artificiales sin conservadores. La tarsorrafia y el colgajo conjuntival son procedimientos efectivos para promover la cicatrización corneal, pero causan una disminución de la visión y no son muy estéticos ${ }^{24}$. Las perforaciones pequeñas, menores de $2 \mathrm{~mm}$, pueden tratarse con la aplicación de adhesivos tisulares ${ }^{25}$. Los defectos mayores requieren una queratoplastia lamelar o penetrante ${ }^{26}$. Las tasas de éxito de estos trasplantes corneales son bajas debido a la pobre cicatrización de la herida y al riesgo persistente de defectos epiteliales a causa de la anestesia corneal. Reed, et al. encontraron que el $75 \%$ de 12 pacientes afectos de cicatriz corneal secundaria a una queratitis por herpes zóster e hipoestesia corneal mantuvieron el injerto corneal claro ${ }^{27}$.

\section{Pronóstico}

La queratitis neurotrófica representa una de las enfermedades oculares más difíciles y desafiantes aún carente de un tratamiento estandarizado. El pronóstico depende de varios factores tales como la causa de la lesión de la sensibilidad corneal, el grado de anestesia o hipoestesia corneal y la asociación con otras enfermedades de la superficie ocular como el ojo seco, la queratitis por exposición y la insuficiencia límbica. Está generalmente aceptado que cuanto más grave es la afección en la sensibilidad corneal, más alta es la probabilidad de progresión de la enfermedad. Es importante recordar al paciente que debe ser revisado frecuentemente, ya que la enfermedad habitualmente carece de signos y síntomas, y que la protección con lágrimas artificiales sin conservadores puede prevenir el defecto epitelial. En el momento actual, el tratamiento de la queratitis neurotrófica es aún conservador, y debe evitarse cualquier procedimiento quirúrgico dirigido a restablecer la transparencia corneal debido al riesgo elevado de defectos epiteliales, úlceras, lisis corneal y perforación tras la cirugía.

\section{Conclusión}

La queratitis neurotrófica es una enfermedad degenerativa corneal que se caracteriza por una disminución en la sensibilidad corneal resultado del daño a la inervación trigeminal. El objetivo del tratamiento es prevenir la extensión del daño y promover una adecuada migración epitelial. El estudio de los neurotransmisores y su aplicación brindan una opción terapéutica al futuro ${ }^{28}$.

\section{Responsabilidades éticas}

Protección de personas y animales. Los autores declaran que para esta investigación no se han realizado experimentos en seres humanos ni en animales.

Confidencialidad de los datos. Los autores declaran que en este artículo no aparecen datos de pacientes.

Derecho a la privacidad y consentimiento informado. Los autores declaran que en este artículo no aparecen datos de pacientes.

\section{Financiamiento}

No se ha recibido más financiación que la propia.

\section{Conflicto de intereses}

Los autores declaran no tener conflictos de intereses.

\section{Bibliografía}

1. Mackie IA. Neuroparalytic keratitis. En: Fraunfelder F, Roy FH, eds. Current ocular therapy. . $^{\text {a }}$ ed. Filadelfia; 1995. p. 506-8.

2. Cobo LM. Corneal complications of herpes zoster ophthalmicus. Prevention and treatment. Cornea. 1988;7(1):50-6.

3. Rosenwasser GO, Holland S, Pflugfelder SC, Lugo M, Heidemann DG, Culbertson WW, et al. Topical anesthetics abuse. Ophthalmology. 1990;97(8):967-72.

4. Varga JH, Subinfeld RS, Wolf TC, Stutzman RD, Peele KA, Clifford WS, et al. Topical anesthetic abuse ring keratitis: Report of four cases. Cornea. 1997;16(4):424-9.

5. Weissman SS, Asbell PA. Effects of topical timolol $(0.5 \%)$ and betaxolol (0.5\%) on corneal sensitivity. Br J Ophthalmol. 1990;74(7):409-12.

6. Szerenyi K, Sorken K, Garbus JJ, Lee M, McDonnell PJ. Decrease in normal human corneal sensitivity with topical diclofenac sodium. Am J Ophthalmol. 1994;118(3):312-5.

7. Hyndiuk RA, Kazarian EL, Schultz RO, Seideman S. Neurotrophic corneal ulcers in diabetes mellitus. Arch Ophthalmol. 1977;95(12):2193-6.

8. Karacorlu MA, Cakiner T, Saylan T. Corneal sensitivity and correlations between decreased sensitivity and anterior segment pathology in ocular leprosy. Br J Ophthalmol. 1991;75(2):117-9.

9. Foster CS. Corneal Manifestations of Neurologic Diseases. En: Smolin G,

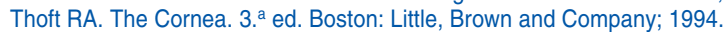

10. Cavanagh HD, Colley AM. The molecular basis of neurotrophic keratitis. Acta Ophthalmol Suppl. 1989;192:115-34.

11. Kingsley RE, Marffurt CF. Topical substance $P$ and corneal epithelia wound closure in the rabbit. Invest Ophthalmol Vis Sci. 1997;38(2):388-95.

12. Chikama T, Fukuda K, Morishige N, Nishida T. Treatment of neurotrophic keratopathy with substance-P-derived peptide (FGLM) and insulin growth factor 1. Lancet. 1998;351(9118):1783-4.

13. Labetoulle M, Auquier P, Conrad H, Crochard A, Daniloski M, Bouée S, et al. Incidence of herpes simplex virus keratitis in France. Ophthalmology. 2005;112(5):888-95. 
Rev Mex Oftalmol. 2018;92

14. Hamrah P, Cruzat A, Dastjerdi MH, Zheng L, Shahatit BM, Bayhan HA et al. Corneal sensation and subbasal nerve alterations in patients with herpes simplex keratitis: an in vivo confocal microscopy study. Ophthalmology. 2010;117(10):1930-6.

15. Dworkin RH, Johnson RW, Breuer J, Gnann JW, Levin MJ, Backonja M et al. Recommendations for the management of herpes zoster. Clin Infect Dis. 2007;44 Suppl 1:S1-26.

16. Bhatti MT, Patel R. Neuro-ophthalmic considerations in trigeminal neuralgia and its surgical treatment. Curr Opin Ophthalmol. 2005;16(6):334-40.

17. Belmonte C, Acosta MC, Schmelz M, Gallar J. Measurement of corneal sensitivity to mechanical and chemical stimulation with a $\mathrm{CO} 2$ esthesiometer. Invest Ophthalmol Vis Sci. 1999;40(2):513-9.

18. Heigle TJ, Pukfelder SC. Aqueous tear production in patients with neurotrophic keratitis. Cornea. 1996;15(2):135-8.

19. Sacchetti M, Lambiase A. Diagnosis and management of neurotrophic keratitis. Clini Ophthalmol. 2014;8:571-9.

20. Sacchetti M, Lambiase A, Cortes M, Sgrulletta R, Bonini S, Merlo D, et al Clinical and cytological findings in limbal stem cell deficiency. Graefes Arch Clin Exp Ophthalmol. 2005;243(9):870-6
21. Grey F, Carley F, Biswas S, Tromans C. Scleral contact lens management of bilateral exposure and neurotrophic keratopathy. Cont Lens Anterior Eye. 2012;35(6):288-91.

22. Lambiase A, Rama $P$, Aloe L, Bonini S. Management of neurotrophic keratopathy. Curr Opin Ophthalmol. 1999;10(4):270-6.

23. Kirkness CM, Adams GGW, Dilly PN, Lee JP. Botulinum toxin A-induced protective ptosis in corneal disease. Ophthalmology. 1988;95(4):473-80.

24. Khodadoust A, Quinter AP. Microsurgical approach to the conjunctival flap. Arch Ophthalmol. 2003:121(8):1189-93.

25. Khokhar S, Natung T, Sony P, Sharma N, Agarwal N, Vajpayee RB. Amniotic membrane transplantation in refractory neurotrophic corneal ulcers: a randomized, controlled clinical trial. Cornea. 2005;24(6):654-60.

26. Jhanji V, Young AL, Mehta JS, Sharma N, Agarwal T, Vajpayee RB. Management of corneal perforation. Surv Ophthalmol. 2011;56(6):522-38.

27. Reed JW, Joyner SJ, Knauer WJ $3^{\text {rd }}$. Penetrating keratoplasty for herpes zoster keratopathy. Am J Ophthalmol. 1989;107(3):257-61.

28. Lambiase A, Sacchetti M, Bonini S. Nerve growth factor therapy for corneal disease. Curr Opin Ophthalmol. 2012;23(4):296-302. 